\title{
Chromomagnetic Instability and Induced Magnetic Field in Neutral Two-Flavor Color Superconductivity
}

\author{
Efrain J. Ferrer and Vivian de la Incera \\ Department of Physics, Western Illinois University, Macomb, IL 61455, USA
}

\begin{abstract}
We find that the chromomagnetic instability existing in neutral two- flavor color superconductivity at moderate densities is removed by the formation of an inhomogeneous condensate of charged gluons and the corresponding induction of a magnetic field. It is shown that this inhomogeneous ground state is energetically favored over a homogeneous one. The spontaneous induction of a magnetic field in a color superconductor at moderate densities can be of interest for the astrophysics of compact stellar objects exhibiting strong magnetic fields as magnetars.

PACS numbers: $12.38 . \mathrm{Aw}, 12.38 .-\mathrm{t}, 24.85 .+\mathrm{p}$
\end{abstract}

\section{INTRODUCTION}

It is quite plausible that color superconductivity (CS) is realized in the cold and dense core of compact stellar objects. For matter densities high enough to guarantee the quark deconfinement, and sufficiently low to consider the decoupling of the strange quark mass $M_{s}$, the quarks can condense to form a two-flavor color superconductor (2SC) [1] . At higher densities, for which $M_{s}$ cannot be regarded as extremely large, the three-flavor phases $g C F L$ 2] and $C F L$ [3] will appear respectively with increasing densities. Matter inside compact stars should be neutral and remain in $\beta$ equilibrium. When these conditions along with $M_{s}$ are taken into account in the dynamics of the gluons in the $g C F L$ phase (or for that matter in the 2SC phase if the s-quark is decoupled), some gluon modes become tachyonic [4, 5], indicating that the system ground state should be restructured.

The quest to find the stable ground state at the realistic, moderate densities of compact stars has led to several interesting proposals. Some of the most promising possibilities are a modified $C F L$-phase with a condensate of kaons [6] that requires a space-dependent condensate to remove the instability; a LOFF phase on which the quarks pair with nonzero total momentum [7]; and a homogenous gluon condensate phase [8]. At present it is not clear if any of these proposals is the final solution to the problem.

In this paper we will show that there is one more possible solution: an inhomogeneous condensate of charged gluons that induces a rotated magnetic field. Our solution is found in the Meissner unstable region of the so-called gapped $2 S C$ phase [9], but we expect that it could also be realized in the three-flavor theory. It has the peculiarity of preserving the electromagnetic gauge invariance $\widetilde{U}_{e m}(1)$ of the color superconductor. As will be shown in our derivations, this inhomogeneous phase is energetically favored over the one with homogeneous gluon condensate and no induced magnetic field. We will also discuss its possible relation to a LOFF-type phase.

In a previous paper [10], we investigated the formation of an inhomogeneous gluon condensate in a color superconductor. However, apart from the fact that in the current work we are going to consider a two-flavor theory, while in Ref. [10] we studied a three-flavor system, there is a fundamental difference between the genesis of the condensation phenomenon that we will discuss in the present paper and the one found in [10]. In Ref. [10] the condensation of gluons was the response of the color superconductor to an externally applied and sufficiently strong magnetic field. In that case, if no magnetic field is present, no gluon condensate is formed. Therefore, the gluon condensation in [10] was a magnetic-field-induced phenomenon. On the other hand, in this work, no external magnetic field is present to begin with. The condensation phenomenon here is connected to a Meissner instability triggered at moderate densities by a pairing stress associated with the neutrality and $\beta$-equilibrium constraints. Such instability occurs in the gapped $2 \mathrm{SC}$ color superconductor at moderate densities when the effective Meissner mass of the charged gluons becomes tachyonic. As we shall show below, in this situation an inhomogeneous condensate of charged gluons emerges to remove the chromomagnetic instability created by the pairing mismatch.

In the case of the gluon condensation produced by a sufficiently strong magnetic field [10], the anomalous magnetic moment interaction of the spin- 1 charged fields with the external magnetic field led to a phenomenon of anti-screening, so that the back reaction of the condensate on the applied field was such that it boosted the inmedium magnetic field strength. This anti-screening is a well-known phenomenon that occurs in all the instances of charged spin-1 field condensation in the presence of a strong magnetic field [11, 12]. Amazingly, as we will show in this paper, the system predisposition to boost an in-medium magnetic field through the magnetic moment of the spin-1 charged condensate works in favor of the spontaneous generation of a rotated magnetic field in the unstable region of the gapped 2SC when no external magnetic field is present. Indeed, in this case the currents associated to the inhomogeneous condensate of charged gluons induce a non-zero rotated magnetic field in the system. Hence, the magnetic field here is a consequence of the gluon condensation, while in [10] it was the other way around. The spontaneous induction of a 
rotated magnetic field in CS systems that have pairings with mismatched Fermi surfaces is a new kind of phenomenon that can serve to generate magnetic fields in stellar compact objects as magnetars.

The plan of the paper is as follows. In Sec Iwe present the effective theory of the gluonic phase with electromagnetic interactions in the neutral two-flavor dense QCD. The arising of an inhomogeneous gluon condensate to remove the chromomagnetic instability caused by the Fermi-surface pairing mismatch in the 2SC color superconductor at moderate densities, as well as the corresponding induction of a rotated magnetic field are investigated in Sec III Finally, in Sec. IV we discuss the significance of the reported findings for the astrophysics of highly magnetized compact objects.

\section{NEUTRAL TWO-FLAVOR DENSE QCD WITH ELECTROMAGNETIC INTERACTIONS}

In the gapped $2 S C$ phase the solution of the neutrality conditions $\partial \Omega_{0} / \partial \mu_{i}=0$, with $\mu_{i}=\mu_{e}, \mu_{8}, \mu_{3}$, and gap equation $\partial \Omega_{0} / \partial \Delta=0$, for the effective potential $\Omega_{0}$ in the mean-field approximation, led to $\mu_{3}=0$, and nonzero values of $\mu_{e}$, and $\mu_{8}$, satisfying $\mu_{8} \ll \mu_{e}<\mu$ for a wide range of parameters [9]. Here $\mu$ is the quark chemical potential, $\mu_{e}$ the electric chemical potential, and the "chemical potentials" $\mu_{8}$ and $\mu_{3}$ are strictly speaking condensates of the time components of gauge fields, $\mu_{8}=(\sqrt{3} g / 2)\left\langle G_{0}^{(8)}\right\rangle$ and $\mu_{3}=(g / 2)\left\langle G_{0}^{(3)}\right\rangle$. The nonzero values of the chemical potentials produce a mismatch between the Fermi spheres of the quark Cooper pairs, $\delta \mu=\mu_{e} / 2$.

The gapped 2SC turned out to be unstable once the gauge fields $\left\{G_{\mu}^{(1)}, G_{\mu}^{(2)}, G_{\mu}^{(3)}, K_{\mu}, K_{\mu}^{\dagger}, \widetilde{G}_{\mu}^{8}, \widetilde{A}_{\mu}\right\}$ were taken into consideration. As shown in Ref. [4], the gluons $G_{\mu}^{(1,2,3)}$ are massless, the in-medium $8^{\text {th }}$-gluon $\widetilde{G}_{\mu}^{8}=$ $\sin \theta A_{\mu}+\cos \theta G_{\mu}^{8}$ has positive Meissner square mass, and the $K$-gluon doublet $K_{\mu}^{\top} \equiv \frac{1}{\sqrt{2}}\left(G_{\mu}^{(4)}-i G_{\mu}^{(5)}, G_{\mu}^{(6)}-i G_{\mu}^{(7)}\right)$ has Meissner square mass that becomes imaginary for $\Delta>\delta \mu>\Delta / \sqrt{2}$, signalizing the onset of an unstable ground state. The mass of the in-medium (rotated) electromagnetic field $\widetilde{A}_{\mu}=\cos \theta A_{\mu}-\sin \theta G_{\mu}^{8}$ is zero, which is consistent with the remaining unbroken $\widetilde{U}(1)_{e m}$ group. Note that even though the gluons are neutral with respect to the regular electric charge, in the 2SC phase the gluon complex doublet $K_{\mu}$ has nonzero rotated charge $\widetilde{Q}=Q-\frac{1}{\sqrt{3}} T_{8}$ of magnitude $\widetilde{q}=\widetilde{e} / 2$, with $\widetilde{e}=e \cos \theta, T_{8}$ the 8 th color generator, and $Q$ the conventional electric charge operator [13].

In what follows, we will find a stable ground state solution near the critical point $\delta \mu_{c}=\Delta / \sqrt{2}$. Close to that point the absolute value of the square magnetic mass $\left|m_{M}^{2}\right|$ is very small, thus allowing analytical calculations. The tachyonic modes in the Meissner-unstable gapped $2 \mathrm{SC}$ are associated only to charged gluon fields. It is nat- ural to expect then that a new, stable ground state should incorporate the condensation of these charged gluons. In general we should allow for an inhomogeneous condensate. Given that this kind of solution may generate rotated electromagnetic currents, the rotated electromagnetism should be included in the general framework of the condensation phenomenon. With this aim, we start from the mean-field effective action of the $2 S C$ gauged NambuJona-Lasinio model depending on the gluon fields, rotated electromagnetic field, diquark condensates, and chemical potentials,

$\Gamma_{e f f}=-\int d^{4} x\left\{\frac{1}{4}\left(f_{\mu \nu}\right)^{2}+\frac{1}{4}\left(F_{\mu \nu}^{a}\right)^{2}\right\}+\Gamma_{g}-\frac{|\Delta|^{2} \beta V}{4 G}+\Gamma_{q}$,

where $a=1, \ldots, 8, G$ is the four-fermion coupling, $\beta$ the inverse temperature, $V$ the 3 -dimensional volume, $f_{\mu \nu}=\partial_{\mu} A_{\nu}-\partial_{\nu} A_{\mu}$ is the conventional electromagnetic strength tensor, $\Gamma_{g}$ is the gauge-fixing action, and $\Gamma_{q}=\frac{1}{2} \operatorname{Tr} \ln \left(S^{-1}+G_{\mu}^{a} \Gamma_{a}^{\mu}+A_{\mu} \Gamma^{\mu}\right)$ is the fermion contribution obtained after integrating out the quark fields in the grand partition function of the neutral $2 S C$ phase [14]. In $\Gamma_{q}, S$ is the quasiparticle propagator in the Nambu-Gor'kov space; $\Gamma_{a}^{\mu}=$ $\operatorname{diag}\left(g \gamma^{\mu} T_{a},-g \gamma^{\mu} T_{a}^{\top}\right)$ is the Nambu-Gor'kov gluon vertex, and $\Gamma^{\mu}=\operatorname{diag}\left(e \gamma^{\mu} Q,-e \gamma^{\mu} Q\right)$ is the Nambu-Gor'kov electromagnetic vertex. In principle, $S$ depends on the diquark condensate, chemical potentials and possible condensates of gauge fields.

We assume a weak-coupling approximation $\left(\alpha_{s}<1\right)$. Then, expanding the logarithm up to second order in the gauge fields, we obtain $\Gamma_{q}=\Gamma_{0}-\frac{1}{2} \int d^{4} x\left[G_{\mu}^{a} \Pi_{a b}^{\mu \nu} G_{\nu}^{b}+\right.$ $\left.A_{\mu} \Pi^{\mu \nu} A_{\nu}\right]$. All the quantities contributing to $\Gamma_{q}$ : the effective action $\left(\Gamma_{0}=\frac{1}{2} T r \ln S^{-1}\right)$, as well as the polarization operators for gluons $\left(\Pi_{a b}^{\mu \nu}=\frac{1}{2} \operatorname{Tr}\left[\Gamma_{a}^{\mu} S \Gamma_{b}^{\nu} S\right]\right)$ and photons $\left(\Pi^{\mu \nu}=\frac{1}{2} \operatorname{Tr}\left[\Gamma^{\mu} S \Gamma^{\nu} S\right]\right)$ have been found in the hard-dense-loop approximation of the neutral 2SC phase [4]. The absence of linear terms in the expansion of $\Gamma_{q}$ is due to the zero trace in the color index, which is a consequence of color neutrality, $\left\langle J^{c}\right\rangle=0$ (i.e. zero tadpole), required to preserve the theory non-Abelian gauge symmetry [15].

In this derivation we used t'Hooft gauge to avoid mixing between the gluons and the fluctuations of the diquark condensate. Details of the implementation of t'Hooft gauge in the 2SC theory can be found in [14]. The scalar modes associated to the fluctuations of the diquark condensate come from the spontaneous breaking of the color gauge group. Therefore, they are unphysical and only serve to provide the longitudinal degrees of freedom of the gluons that acquire a mass due to the symmetry breaking. The decoupling of the scalar modes from the gluons occurs in the t'Hooft gauge for arbitrary values of the gauge parameter $\lambda$. Later on we will choose $\lambda=1$ because it simplifies the calculations in the gluon sector. As always, physical parameters as Debye and Meissner masses do not depend on $\lambda$. 


\section{INHOMOGENEOUS GLUON CONDENSATE AND INDUCED IN-MEDIUM MAGNETIC FIELD}

To investigate the condensation phenomenon triggered by the tachyonic modes of the charged gluons, we can restrict our analysis to the gauge sector of the mean-field effective action (1) that depends on the charged gluon fields and rotated electromagnetic field. For a static solution, one only needs the leading contribution of the polarization operators in the infrared limit $\left(p_{0}=0,|\vec{p}| \rightarrow 0\right)$. Under these conditions, the gauge sector of the effective action can be written as

$$
\begin{aligned}
\Gamma_{e f f}^{g} & =\int d^{4} x\left\{-\frac{1}{4}\left(\widetilde{f}_{\mu \nu}\right)^{2}-\frac{1}{2}\left|\widetilde{\Pi}_{\mu} K_{\nu}-\widetilde{\Pi}_{\nu} K_{\mu}\right|^{2}\right. \\
& -\left[m_{M}^{2} \delta_{\mu i} \delta_{\nu i}+\left(\mu_{8}-\mu_{3}\right)^{2} \delta_{\mu \nu}+i \widetilde{q} \widetilde{f}_{\mu \nu}\right] K_{\mu} K_{\nu}^{\dagger} \\
& \left.+\frac{g^{2}}{2}\left[\left(K_{\mu}\right)^{2}\left(K_{\nu}^{\dagger}\right)^{2}-\left(K_{\mu} K_{\mu}^{\dagger}\right)^{2}\right]+\frac{1}{\lambda} K_{\mu}^{\dagger} \widetilde{\Pi}_{\mu} \widetilde{\Pi}_{\nu} K_{\nu}\right\}
\end{aligned}
$$

where $m_{M}^{2}=\frac{2 \alpha_{s} \bar{\mu}^{2}}{3 \pi}\left[1-\frac{2 \delta \mu^{2}}{\Delta^{2}}\right]$, is the Meissner mass with $\bar{\mu}=\mu-\frac{\mu_{e}}{6}+\frac{\mu_{8}}{3}$ and $\alpha_{s} \equiv \frac{g^{2}}{4 \pi}$ [4], the last term in (2) comes from the $\Gamma_{g}$ term in (1), taken in the 't Hooft gauge with gauge-fixing parameter $\lambda, \widetilde{\Pi}_{\mu}=\partial_{\mu}-i \widetilde{q} \widetilde{A}_{\mu}$ and $\widetilde{f}_{\mu \nu}=\partial_{\mu} \widetilde{A}_{\nu}-\partial_{\nu} \widetilde{A}_{\mu}$. In (2) the Debye mass $m_{D}$ was not included since it will be no substantial for our derivations. The chemical potential $\mu_{3}$, although is zero in the gapped phase, should be in principle taken into account in the analysis of the new phase, since the $\mathrm{K}$ condensate will break the remaining $S U(2)_{C}$ symmetry.

At this point let us consider for a moment that we have an external rotated magnetic field $\widetilde{H}$. In this case the effective action (2) becomes that of a spin-1 charged field in a magnetic field (see for instance [16]). If we work in the region where $m_{M}^{2}-\mu_{8}^{2}>0$, the ground state is stable $\left(\mu_{3}=0\right.$ in the stable region). Under these circumstances, we know [10] that when $q \widetilde{H} \geq \widetilde{q} \widetilde{H}_{c}=m_{M}^{2}-\mu_{8}^{2}$, the effective magnetic mass of one of the charged gluon modes becomes imaginary due to the anomalous magnetic moment term $i \widetilde{q} \widetilde{f}_{\mu \nu} K_{\mu} K_{\nu}^{\dagger}$. This field-induced instability triggers the formation of a gluon-vortex state characterized by the antiscreening of the magnetic field.

Now, let us go back to the situation of interest in the present work, that is, a system with no external magnetic field. As usual in theories with zero-component gaugefield condensates [17], $\mu_{8}$ gives rise to a tachyonic mass contribution. This tachyonic contribution creates a region of instability in a density interval for which $m_{M}^{2}$ is still positive. Let us elaborate on this point. Using the results of [4], one can easily realize that when the density is decreasing, coming from values where $\delta \mu<\Delta / \sqrt{2}$, the different chemical potentials accordingly change in such a way that the pairing mismatch $\delta \mu$ increases. Consequently, the Meissner mass $m_{M}^{2}$ decreases and, at some density value, it crosses the line of $\mu_{8}$, leading to a tachyonic effective Meissner mass $\left(m_{M}^{2}-\mu_{8}^{2}<0\right)$ for the range of densities between the crossing point and the value where $\delta \mu=\delta \mu_{c}$, even though $m_{M}^{2}>0$ in this interval. Once $\delta \mu \geq \delta \mu_{c}$, the Meissner mass square $m_{M}^{2}$ becomes negative itself, so the two terms of the effective Meissner mass give tachyonic contributions. Although the instability in the region where $m_{M}^{2}>0$ is a direct consequence of the pairing mismatch, it formally resembles the instability produced by an external magnetic field on an otherwise stable color superconductor [10]. The formal role of the magnetic field is played here by $\mu_{8}$. Borrowing from the experience gained in the case with external magnetic field, we would expect that this Meissner instability will also be removed through the spontaneous generation of an inhomogeneous gluon condensate $\left\langle K_{i}\right\rangle$ capable to induce a rotated magnetic field, thanks to the anomalous magnetic moment of the spin-1 charged particles. Having this in mind, we propose the following ansatz

$$
\left\langle K_{\mu}\right\rangle \equiv \frac{1}{\sqrt{2}}\left(\begin{array}{c}
\bar{G}_{\mu} \\
0
\end{array}\right), \quad \bar{G}_{\mu} \equiv \bar{G}(x, y)(0,1,-i, 0)
$$

where we took advantage of the $S U(2)_{c}$ symmetry to write the $\left\langle K_{i}\right\rangle$-doublet with only one nonzero component. Since in this ansatz the inhomogeneity of the gluon condensate is taken in the $(x, y)$-plane, it follows that the corresponding induced magnetic field will be aligned in the perpendicular direction, i.e. along the z-axes, $\left\langle\widetilde{f}_{12}\right\rangle=\widetilde{B}$. The part of the free energy density that depends on the gauge-field condensates, $\mathcal{F}_{g}=\mathcal{F}-\mathcal{F}_{n 0}$, with $\mathcal{F}_{n 0}=$ $-\Gamma_{0}=\Omega_{0}$ denoting the system free-energy density in the absence of the gauge-field condensate $(\bar{G}=0, \widetilde{B}=0)$, is found, after fixing the gauge parameter to $\lambda=1$ and using the ansatz (3) in (2), to be

$$
\begin{aligned}
\mathcal{F}_{g} & =\frac{\widetilde{B}^{2}}{2}-2 \bar{G}^{*} \widetilde{\Pi}^{2} \bar{G}+2 g^{2}|\bar{G}|^{4} \\
& -2\left[2 \widetilde{q} \widetilde{B}+\left(\mu_{3}+\mu_{8}\right)^{2}+m_{M}^{2}\right]|\bar{G}|^{2}
\end{aligned}
$$

From the neutrality condition for the $3^{r d}$-color charge it is found that $\mu_{3}=\mu_{8}$. The fact that $\mu_{3}$ gets a finite value just after the critical point $m_{M}^{2}-\mu_{8}^{2}=0$ is an indication of a first-order phase transition, but since $\mu_{8}$ is parametrically suppressed in the gapped phase by the quark chemical potential $\mu_{8} \sim \Delta^{2} / \mu$ [4], it will be a weakly first-order phase transition. Henceforth, we will consider that $\mu_{3}=\mu_{8}$ in (4), and work close to the transition point $\delta \mu \gtrsim \delta \mu_{c}$ which is the point where $m_{M}^{2}$ continuously changes sign to a negative value. For very small, negative values of $m_{M}^{2}$, the gluon condensate and the induced magnetic field should be very small too, thereby facilitating the calculations.

Minimizing (4) with respect to $\bar{G}^{*}$ gives

$$
-\widetilde{\Pi}^{2} \bar{G}-\left(2 \widetilde{q} \widetilde{B}+m_{M}^{2}\right) \bar{G}+2 g^{2}|\bar{G}|^{2} \bar{G}=0
$$

Eq. (5) is a highly non-linear differential equation that can be exactly solved only by numerical methods. Nevertheless, we can take advantage of working near the transition point, where we can manage to find an approximated 
solution that will lead to a qualitative understanding of the new condensate phase. With this aim, and guided by the experience with external fields, where the solution is always such that the kinetic term $\left|\widetilde{\Pi}_{\mu} K_{\nu}-\widetilde{\Pi}_{\nu} K_{\mu}\right|^{2}$ is approximately zero near the transition point, we will consider that when $\delta \mu \simeq \delta \mu_{c}$ our solution will satisfy the same condition. Hence, we will look for a minimum solution satisfying

$$
\widetilde{\Pi}^{2} \bar{G}+\widetilde{q} \widetilde{B} \bar{G} \simeq 0 .
$$

With the help of (6) one can show that the minimum equation for the induced field $\widetilde{B}$ takes the form

$$
2 \widetilde{q}|\bar{G}|^{2}-\widetilde{B} \simeq 0
$$

The relative sign between the two terms in Eq. (7) implies that for $|\bar{G}| \neq 0$ a magnetic field $\widetilde{B}$ is induced. The origin of that possibility can be traced back to the anomalous magnetic moment term in the action of the charged gluons. This effect has the same physical root as the paramagnetism found in Ref. [10]; where contrary to what occurs in conventional superconductivity, the resultant in-medium field $\widetilde{B}$ becomes stronger than the applied field $\widetilde{H}$ that triggers the instability. The antiscreening of a magnetic field by the condensation of charged spin- 1 fields has been also found in the context of the electroweak theory [12].

Using the minimum equations (5) and (7) in (4), we obtain the condensation free-energy density

$$
\overline{\mathcal{F}}_{g} \simeq-2\left(g^{2}-\widetilde{q}^{2}\right)|\bar{G}|^{4}
$$

The hierarchy between the strong $(g)$ and the electromagnetic $(\widetilde{q})$ couplings implies that $\overline{\mathcal{F}}_{c}<0$. Therefore, although the induction of a magnetic field always costs energy (as can be seen from the positive first term in (4)), the field interaction with the gluon anomalous magnetic moment, produces a sufficiently large negative contribution to compensate for that increase. Consequently, as seen from (88), the net effect of the proposed condensates is to decrease the system free-energy density.

It follows from Eqs.(5)-(17) that near the phase transition point the inhomogeneity of the condensate solution should be a small but nonzero correction to a leading constant term

$$
\begin{gathered}
|\bar{G}|^{2} \simeq \Lambda_{g / \widetilde{q}} m_{M}^{2} / 2 \widetilde{q}^{2}+\mathcal{O}\left(m_{M}^{4}\right) f(x, y), \\
\widetilde{q} \widetilde{B} \simeq \Lambda_{g / \widetilde{q}} m_{M}^{2}+\mathcal{O}\left(m_{M}^{4}\right) g(x, y) .
\end{gathered}
$$

with $\Lambda_{g / \widetilde{q}} \equiv\left(g^{2} / \widetilde{q}^{2}-1\right)^{-1}$.

The explicit form of the inhomogeneity can be found from (6), which can be written in polar coordinates as

$$
\left[\frac{1}{r} \partial_{r}\left(r \partial_{r}\right)+\frac{1}{r^{2}} \partial_{\theta}^{2}+\frac{1}{\xi^{2}}\left(1-i \partial_{\theta}\right)-\frac{r^{2}}{4 \xi^{4}}\right] G(r, \theta)=0
$$

In the above equation we approached the rotated magnetic field by its leading in (10), used the symmetric gauge $\widetilde{A}_{i}=-(\widetilde{B} / 2) \epsilon_{i j} x_{j}$, and introduced the characteristic length $\xi^{2} \equiv 1 / \Lambda_{g / \widetilde{q}} m_{M}^{2}$. Using just the leading contribution of $\widetilde{B}$ in (11) is a consistent approximation if we simultaneously drop the $\frac{r^{2}}{4 \xi^{4}}$ term and restrict the solution to the domain $r \ll \xi$. Notice that this domain is indeed a large region because near the critical point the magnitude of $m_{M}$ is very small, hence $\xi$ becomes very large. The most symmetric solution of Eq.(11) is the one that preserves the $\mathrm{SO}(2)$ symmetry in the $(x, y)$ plane. Hence, proposing a solution of the form $G(r, \theta) \sim R(r) e^{i \chi}$, with $\chi$ a constant phase, the equation for $R(r)$ reduces to $\left[r \partial_{r}\left(r \partial_{r}\right)+\frac{r^{2}}{\xi^{2}}\right] R(r)=0$. It is solved by the Bessel function of the first kind $J_{0}(r / \xi)$. Then, the gluon condensate can be written as $G(r)=(1 / \sqrt{2} \widetilde{q} \xi) J_{0}(r / \xi) \exp i \chi$, which is consistent with (9), as in the domain of validity of this solution $(r \ll \xi)$ the series can be approximated by its first terms. Accordingly, the modulus of the condensate square satisfies

$$
|\bar{G}|^{2} \simeq \frac{1}{2 \widetilde{q}^{2} \xi^{2}}-\frac{r^{2}}{4 \widetilde{q}^{2} \xi^{4}}
$$

The improved solution for $\widetilde{B}$ is found substituting (12) back into (7). The induced field $\widetilde{B}$ is homogeneous in the $z$-direction and inhomogeneous in the $(x, y)$-plane.

As always, the choosing of a particular gauge condition $(\lambda=1$ in this case) is dictated by convenience. As occurs in other gauge theories, the particular form of the condensate solution $G(r, \theta)$ and the effective action (or the effective potential in the case of constant condensates) are gauge-dependent. There is nothing wrong in that, as none of these quantities is physically measurable. What is gauge independent is the effective action evaluated in the minimum solution. Therefore, if the result (12) is plugged back into (8), one obtains a gaugeindependent quantity representing the free-energy density of the ground state.

One may wonder whether this inhomogeneous gluon condensate forms a vortex state. To answer this question we can compare our results with the case with external magnetic field [10]. For this we should notice that the mathematical problem we have just solved is formally similar to that where the instability is induced by a weak external field. This would be the situation when the $2 S C$ system approaches the transition point from the stable side (real effective magnetic mass) and the external magnetic field is slightly larger than a very small but still positive effective mass square $\widetilde{H} \gtrsim \widetilde{H}_{c}=m_{M}^{2}-\mu_{8}^{2} \ll 1$. We know that at large $m_{M}^{2}-\mu_{8}^{2}$ the condensate solution is a crystalline array of vortex-cells with cell's size $\sim \xi \ll 1$. At smaller $m_{M}^{2}-\mu_{8}^{2}$ the lattice structure should remain, but with a larger separation between cells, since in this case $\xi \gg 1$. However, the use of a linear approximation to solve the equations in this case only allows to explore the solution inside an individual cell $(r \ll \xi)$. This is 
the same limitation we have in the linear approach followed in the present paper. Therefore, we expect that when the nonlinear equations will be solved, the vortex arrangement will be explicitly manifested.

The idea of removing the $2 S C$ chromomagnetic instability with the help of a gluon condensate has been previously considered in Ref. [8]. In that work a homogeneous gluon condensate with no rotated magnetic field was proposed. Near the transition point the homogeneous condensate produces a contribution to the condensation energy density that can be approximated as $\overline{\mathcal{F}}_{g} \simeq-\frac{\pi}{2 \alpha^{3}}\left(m_{M}^{2}\right)^{3} / m_{g}^{2}$, with $m_{g}^{2}=4 \alpha_{s}^{2} \bar{\mu}^{2} / 3 \pi$. In this case, the condensation energy in a circle of radius $c \xi$, with $c$ being a dimensionless constant satisfying $c<1$, is $\bar{F}_{g} \simeq-\frac{c^{2} \pi^{2}}{2 \alpha_{s}^{3} \Lambda_{g / \widetilde{q}}} \frac{m_{M}^{4}}{m_{g}^{2}}$. On the other hand, for the $G-\widetilde{B}$ condensate considered in the present paper, the corresponding condensation energy is $\bar{F}_{g} \simeq-c^{2} \pi m_{M}^{2} / 2 \widetilde{q}^{2}$, which is found integrating the free-energy density (8) evaluated in the solution (12) in the circle of radius $c \xi$. Taking into account that near the transition point $m_{M}^{2} \ll m_{g}^{2}[4]$, one can see that the inhomogeneous gluon condensate is energetically favored over the homogeneous one.

We should stress that as $\delta \mu-\delta \mu_{c}$ becomes much larger, the contribution of $\bar{G}$ and $\widetilde{B}$ in the quasiparticle propagator $S$ cannot be neglected, thereby affecting the gap equation (as shown in [18], a sufficiently strong magnetic field can qualitatively influence the gap). As a consequence, the inhomogeneity of the gluon condensate will be naturally transferred to the diquark condensate. Hence, a LOFF-type phase may appear as a back reaction of the inhomogeneous gluon condensate on the gap solution. It is natural to expect that the additional reduction in freeenergy due to the $G-\widetilde{B}$ condensation will make this phase energetically favored over a pure LOFF one [7].

We anticipate that a $G-\widetilde{B}$ condensate will likely remove the chromomagnetic instability in $g C F L$ too. In the gCFL case there are four gluons with tachyonic masses. Following the results of the last paper in Ref. [5], the four tachyonic gluons are $A_{1}, A_{2}$ and two combinations of $A_{3}, A_{8}$ and $A_{\gamma}$. A third combination of $A_{3}$, $A_{8}$ and $A_{\gamma}$ is massless, hence it represents the rotated electromagnetic field in the gCFL phase. Notice that, in the unstable gCFL phase the gluons $A_{1}$ and $A_{2}$ acquire rotated charge since they couple to the new rotated electromagnetic field through its $A_{3}$ component. This implies that $A_{1}$ and $A_{2}$ are analogous to the charged gluons that become tachyonic in the $2 \mathrm{SC}$ case. If $A_{1}$ and $A_{2}$ condense in an inhomogeneous condensate following the same mechanism we found in the present paper, this condensate could induce a rotated magnetic field and also give real masses to the two combinations of $A_{3}, A_{8}$ and $A_{\gamma}$ that were tachyonic. Of course, it is possible too that in addition to the condensation of the charged gluons, the other two tachyonic combinations also condense in the gCFL case. The only way to find out which scenario is the most energetically favored is by doing explicit calculations. However, exploring this idea will be more challenging, as one will have to deal with a strongly firstorder phase transition.

\section{ASTROPHYSICAL CONNOTATIONS}

A common characteristic of neutron stars is their strong magnetization. Their surface magnetic fields range from $B=1.7 \times 10^{8} G$ (PSR B1957+20) up to $2.1 \times 10^{13} G$ (PSR B0154+61), with a typical value of $10^{12} G$ [19]. There are observational discoveries of even strongly magnetized stellar objects- known as magnetarswith surface magnetic fields of order $B \sim 10^{14}-10^{16} G$ [20]. In the core of these compact objects, the field may be considerably larger due to flux conservation during the core collapse. By applying the scalar virial theorem it can be shown that the interior field can reach values of order $B \sim 10^{18} G[21]$.

The observed stellar magnetic fields are supposed to be created by a magnetohydrodynamic dynamo mechanism that amplifies a seed magnetic field due to a rapidly rotating protoneutron star. Thus, the standard explanation of the origin of the magnetar's large magnetic field implies that the rotation should have a spin period $<3 \mathrm{~ms}$. Nevertheless, this mechanism cannot explain all the features of the supernova remnants surrounding these objects [22].

It has been found [4] that when $\delta \mu=\Delta$ in the neutral 2SC model, the absolute value of the magnetic mass becomes of order $m_{g}$. This implies that the inhomogeneous gluon condensate found in our paper could produce a magnetic field of order of magnitude in the range $\sim 10^{16}-10^{17} \mathrm{G}$. As discussed in Refs. [23], the possibility of generating a magnetic field of such a large magnitude in the core of a compact star, without relying on a magneto-hydrodynamics effect, can be an interesting alternative to address the main criticism [22] to the observational conundrum of the standard magnetar's paradigm [20]. On the other hand, to have a mechanism that associates the existence of high magnetic fields to CS at moderate densities can serve to single out the magnetars as the most probable astronomical objects for the realization of this high-dense state of matter.

Acknowledgments: We are grateful to M. Alford, L. McLerran, V. A. Miransky and I. A. Shovkovy for insightful discussions and comments. This work has been supported in part by DOE Nuclear Theory grant DEFG02-07ER41458. 
Lett. 92, 222001 (2004).

[3] M. Alford, K. Rajagopal and F. Wilczek, Phys. Lett. B 422, 247 (1998); Nucl. Phys. B 537, 443 (1999); R. Rapp, T. Schafer, E. V. Shuryak, and M. Velkovsky, Phys. Rev. Lett. 8153 (1998).

[4] M. Huang and I. A. Shovkovy, Phys. Rev. D 70, 051501 (2004); 70, 094030 (2004).

[5] R. Casalbuoni, et. al. Phys. Lett. B 605, 362 (2005); 615, 297(E) (2005); M. Alford and Q. H. Wang, J. Phys. G 31, 719 (2005); K. Fukushima, Phys. Rev. D 72, 074002 (2005).

[6] P. F. Bedaque and T. Schafer, Nucl. Phys. A 697, 802 (2002); A. Kryjevski and T. Schafer, Phys. Lett. B 606, 52 (2005); A. Gerhold and T. Schafer, Phys. Rev. D 73, 125022 (2006).

[7] M. Alford, J. A. Bowers, and K. Rajagopal, Phys. Rev. D. 63, 074016 (2001); I. Giannakis and H.C. Ren, Phys. Lett. B 611, 137 (2005); Nucl. Phys. B 723, 255 (2005); R. Casalbuoni, et.al, Phys. Lett. B 627, 89 (2005); M. Ciminale, G. Nardulli, M. Ruggieri and R. Gatto, Phys. Lett. B 636, 317 (2006); K. Rajagopal and R. Sharma, Phys. Rev. D 74, 094019 (2006).

[8] E. V. Gorbar, M Hashimoto, and V. A. Miransky, Phys. Lett. B 632, 305 (2006); hep-ph/0701211.

[9] I. Shovkovy and M. Huang, Phys. Lett. B 564, 205 (2003); Nucl. Phys. A 729, 835 (2003).

[10] E. J. Ferrer, and V. de la Incera, Phys. Rev. Lett. 97, 122301 (2006); J. Phys. A: Math. Theor. 40, 6913 (2007).

[11] V. V. Skalozub, Sov. J. Nucl. Phys. 28, 113 (1978); N. K. Nielsen and P. Olesen, Nucl. Phys. B 144, 376 (1978); V. V. Skalozub, Sov. J. Nucl. Phys. 43, 665 (1986); 45, 1058 (1987); S. Ferrara and M. Porrati, Mod. Phys. Lett. A 8, 2497 (1993); E. J. Ferrer and V. de la Incera, Int. Jour. of Mod. Phys. A 11, 3875 (1996).

[12] J. Ambjorn and P. Olesen, Nucl. Phys. B 315, 606
(1989); Phys. Lett. B 218, 67 (1989).

[13] D. Litim and C. Manuel, Phys. Rev. D 64, 094013 (2001).

[14] D. H. Rischke, and I. A. Shovkovy, Phys. Rev. D 66, 054019 (2002).

[15] E. J. Ferrer, V. de la Incera, and A.E. Shabad, Nuovo Cim. A 98, 245 (1987).

[16] E. Elizalde and E. J. Ferrer, and V. de la Incera, Ann. of Phys. 295, 33 (2002); Phys. Rev. D 70, 043012 (2004).

[17] A. D. Linde, Phys. Lett. B 8639 (1979); E. J. Ferrer, V. de la Incera and A. E. Shabad, Phys. Lett. B 185407 (1987); Nucl. Phys. B 309120 (1988).

[18] E. J. Ferrer, V. de la Incera and C. Manuel, Phys. Rev. Lett. 95, 152002 (2005); Nucl. Phys. B 747, 88 (2006).

[19] I. Fushiki, E. H. Gudmundsson and C. J. Pethick, Astrophys. J. 342, 958 (1989); T. A. Mihara, et al., Nature (London) 346, 250 (1990); G. Chanmugam, Ann. Rev. Astron. Astrophys. 30, 143 (1992); J. H. Taylor, R. N. Manchester and A. G. Lyne, Astrophys. J. S. 88, 529 (1993); P. P. Kronberg, Rep. Prog. Phys. 57, 325 (1994); D. Lai Rev. Mod. Phys. 73, 629 (2001); D. Grasso and H. R. Rubinstein Phys. Rep. 348, 163 (2001).

[20] C. Thompson and R. C. Duncan, Astrophys. J. 392, L9 (1992); 473, 322 (1996); S. Kulkarni and D. Frail, Nature 365, 33 (1993); T. Murakami et al., Nature 368, 127 (1994); Ibrahim et al., Astrophys. J. 609, L21 (2004).

[21] L. Dong and S. L. Shapiro Astrophys. J. 383, 745 (1991).

[22] J. Vink and L. Kuiper, Mon. Not. Roy. Astron. Soc. Lett. 370 (2006) L14; R-X Xu, astro-ph/0611608

[23] E. J. Ferrer, and V. de la Incera, Phys. Rev. D 76, 045011 (2007) (nucl-th/0703034); E. J. Ferrer, arXiv:0705.2403 [hep-ph] (To appear in the AIP Proceedings of the VII Latin American Symposium on Nuclear Physics and Applications.) 